\title{
Characterization of how contaminants arise in a dredged marine sediment and analysis of the effect of natural weathering
}

\section{Julien Couvidat $1^{\text {a }}$}

Vincent Chatain $1^{\mathrm{a}}$ * *

Vincent.Chatain@insa-lyon.fr

Hassan

Mostafa Benzaazouata $z^{\underline{a}}$

aㅡ-Université de Lyon, INSA Lyon, Laboratoire Déchets Eaux Environnement Pollutions (DEEP) - EA 7429, 7 rue de la Physique, e69621 Villeurbanne Cedex, France

${ }^{25}$ ULQAT-Polytechnique, fInstitut de Recherche en Mines et Environnement (IRME), 445 Boul. de l':-Université, Rouyn-Noranda J9X 5E4, Canada

${ }^{3}$-Université de Liège (ULG), Laboratoire de Génie Minéral, Matériaux et Environnement (GeMMe), Allée de la découverte, 13/A. Bât. B52/3 Sart-Tilman, 4000 Liège, Belgium.

${ }^{*}$ Corresponding author

\section{Abstract}

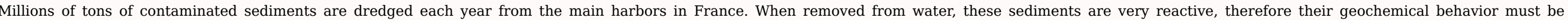

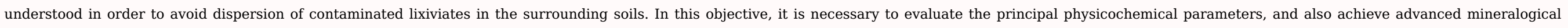

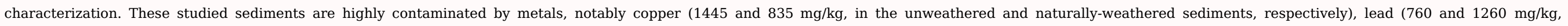

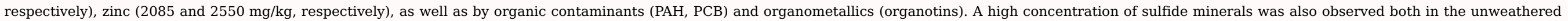
sediment preserved under water ( $3.4 \mathrm{wt}+\%$ of pyrite especially), and in the naturally weathered sediment ( $2 \mathrm{wt}-\%$ pyrite), and in particular framboïdal pyrite was observed in the two materials.

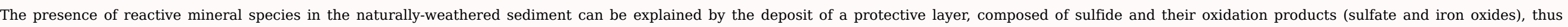

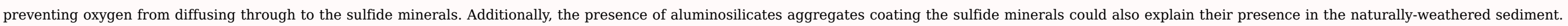

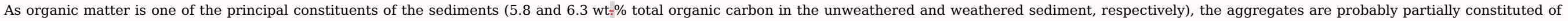

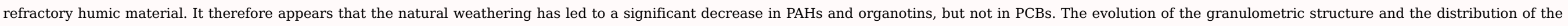
metallic contaminants could therefore lead us to consider a treatment by size separation, and a possible valorization of the dredged sediments in civil engineering.

Keywords: Biogeochemical reactivity; Contaminated marine sediment; Sulfides; Leaching; $\ominus$ Oxidation; Organic matter

\subsection{Introduction}

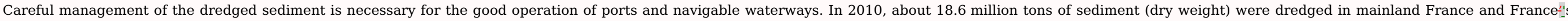

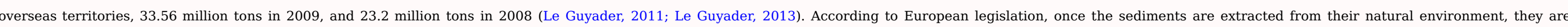
considered as waste and thus must be consequently managed according to the legislation in force (Commission Decision of 3 May 2000 Replacing Decision 94/3/EC Establishing a List of Wastes, 2002: JORF, 2007).

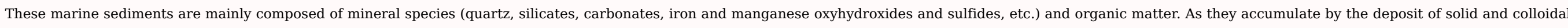

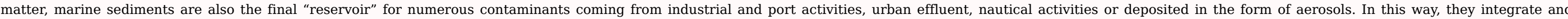

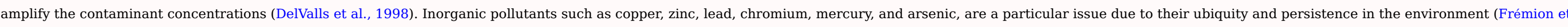

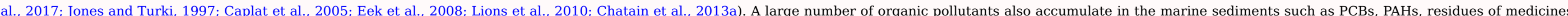




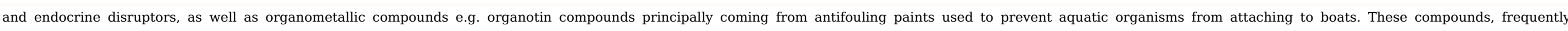

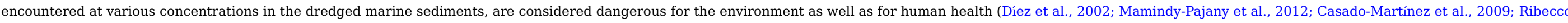
et al., 2011; Staniszewska et al., 2011).

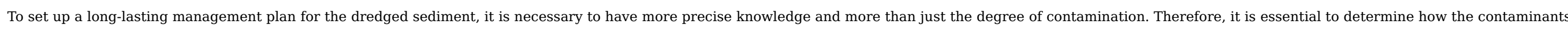

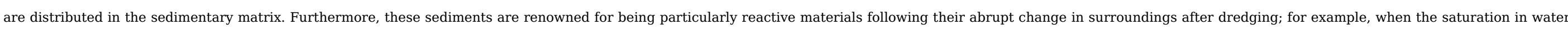

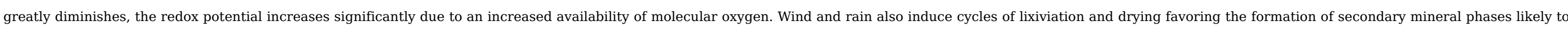

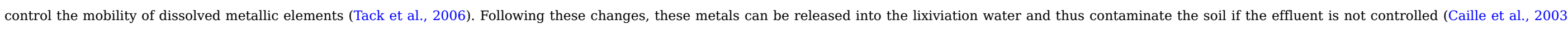

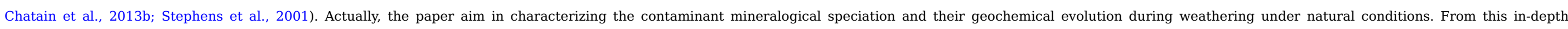
characterization, adapted management solutions could be proposed and evaluated.

\subsection{Material and methods}

\subsubsection{Sampling, storage and treatment}

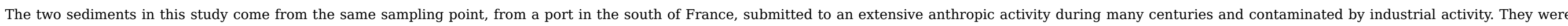

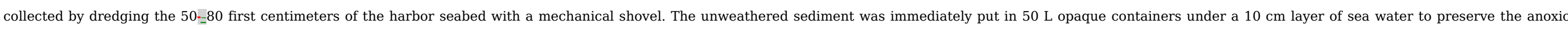

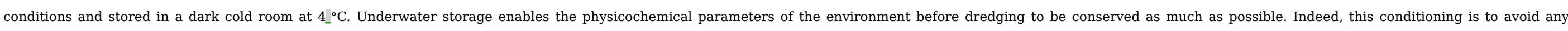

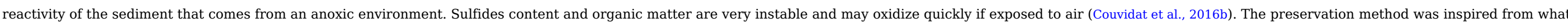
recommended during sulfides mine waste handling (Benzaazoua et al., 1999).

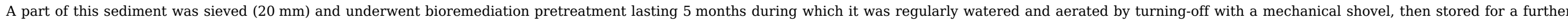

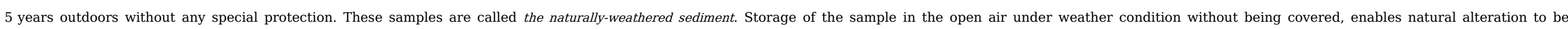

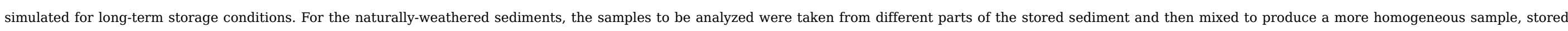
also in the dark at $4{ }_{-}^{\circ} \mathrm{C}$.

\section{3 Characterization of dredged sediments 3.1.3.1 Physicochemical parameters}

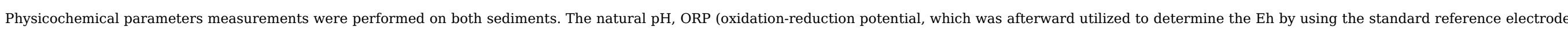

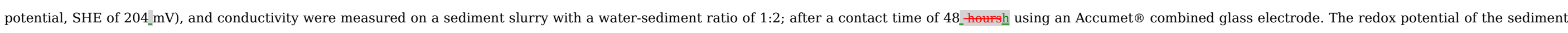

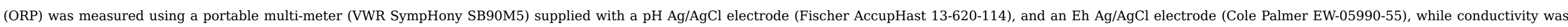

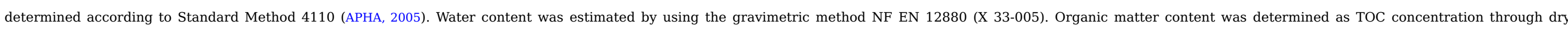

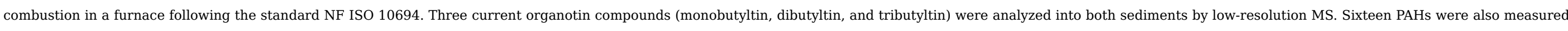

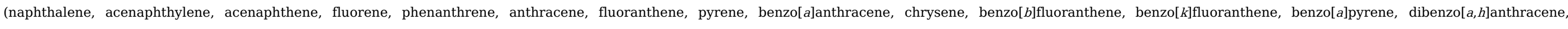

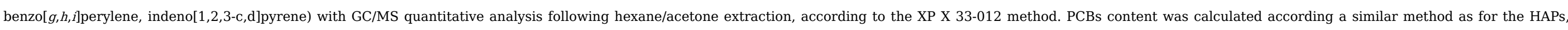
represented by the sum of 7 regulated PCB species (PCB 28, 52, 101, 118, 138, 153, and 180).

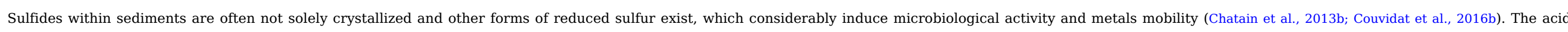

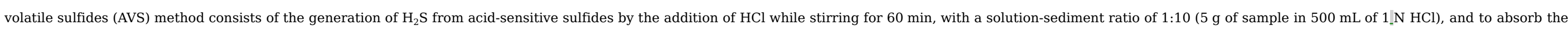

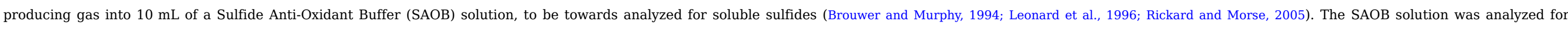
dissolved sulfides by spectrophotometry (HACH DR-890 Colorimeter) using the methylene blue method (equivalent procedure to USEPA 376.2 or Standard Method 4500-S2-D for wastewater). 


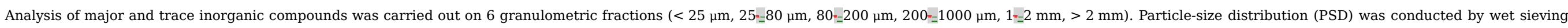

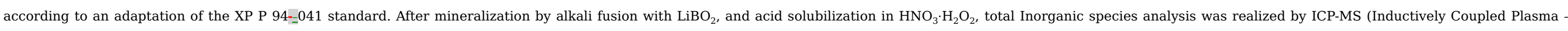
Mass Spectroscopy) accordingly to the internal procedure of the SARM laboratory (Carignan et al., 2001).

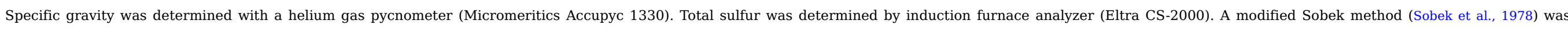

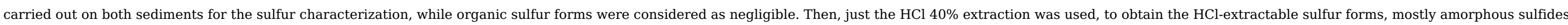

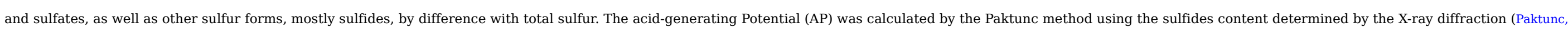
1999a; Paktunc, 1999b), and the neutralization potential (NP) was determined by the Sobek et al. (1978) method modified by Lawrence and Wang (1997)(Lawrenee and Wang, 1997, Sobek et al., 1978).

\subsubsection{Mineralogical analysis}

The mineralogy of both sediments was carefully unalysedanalyzed using Optical Microscopy (OM), X-Ray Diffraction (XRD) and Scanning Electron Microscopy (SEM).

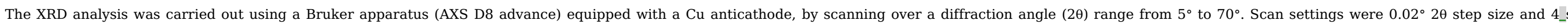

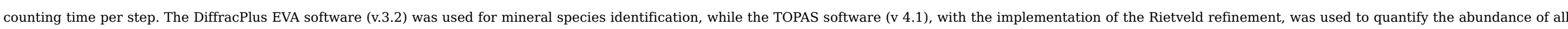

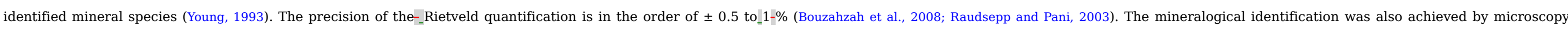

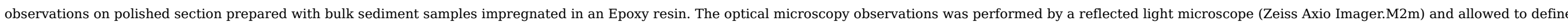

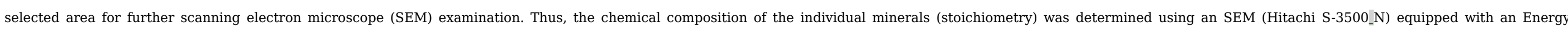
Dispersive Spectrometer (EDS, Silicon Drift Detector X-Max $20 \mathrm{~mm}^{2}$, Oxford) operated under the INCA software (450 Energy). The operating conditions were $20 \mathrm{keV}$, $\sim 100 \mu \mathrm{A}$ and $15 \mathrm{~mm}$ working distance.

\subsubsection{Kinetic test: weathering cells}

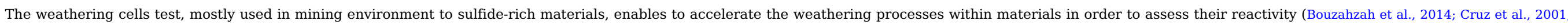
Villeneuve et al., 2009).

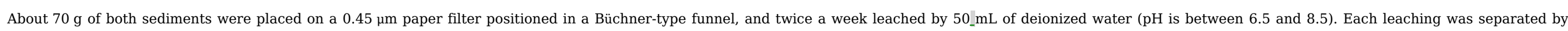

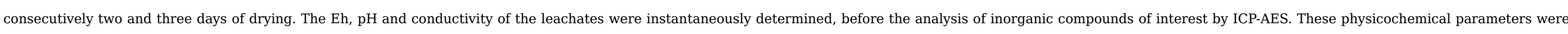
realized over 18 cycles, until reaching a geochemical equilibrium of the leachates.

\section{4 Results}

\subsubsection{Physicochemical characterization}

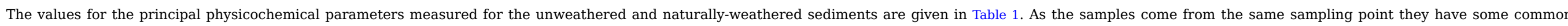

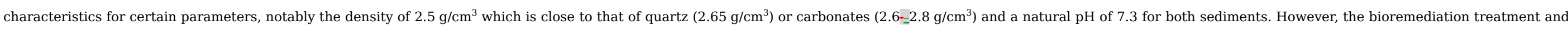

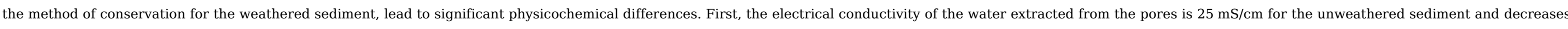

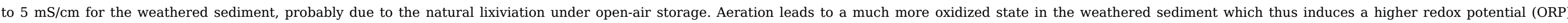

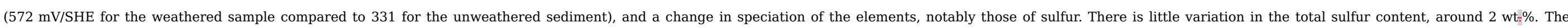

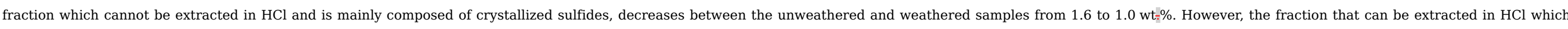

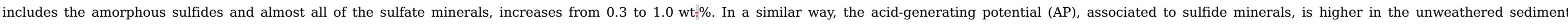

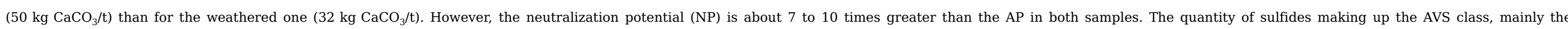

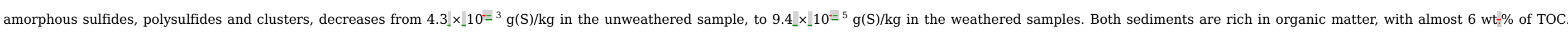

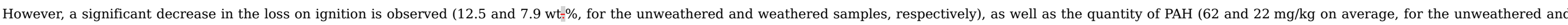




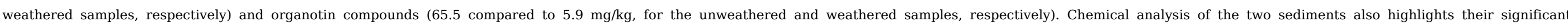

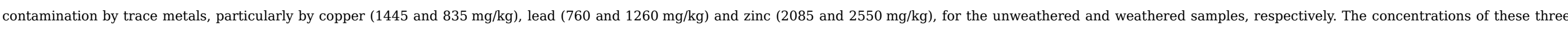

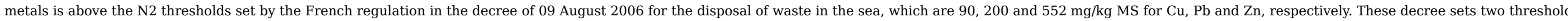

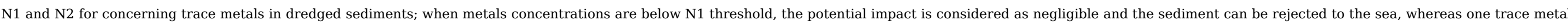

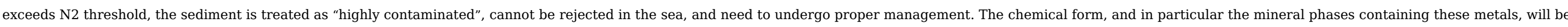
discussed with the mineralogical results.

Table 1 Physicochemical characteristics of the sediments.

alt-text: Table 1

\begin{tabular}{|c|c|c|c|}
\hline Parameter & Unit & Unweathered & Weathered \\
\hline $\mathrm{pH}$ & $=$ & 7.4 & 7.3 \\
\hline ORP & $\mathrm{mV} / \mathrm{SHE}$ & 331 & 572 \\
\hline Conductivity & $\mathrm{mS} / \mathrm{cm}$ & 25 & 5 \\
\hline Water content & wt:\% & 47 & 23 \\
\hline Loss on ignition & $w t-\%$ & 12.5 & 7.9 \\
\hline TOC & $w t=\%$ & 5.8 & 6.3 \\
\hline$\sum$ PAHs & $\mathrm{mg} / \mathrm{kg}$ & $62.18_{-}<\mathrm{x}_{-}<62.4$ & $21.93<<\mathrm{x}_{-}<22.35$ \\
\hline$\sum$ PCBs & $\mathrm{mg} / \mathrm{kg}$ & $0.96 \_<x_{-}<-0.97$ & $1.07 \_<\mathrm{x}_{-}<-1.21$ \\
\hline$\sum$ e्Organotins & $\mathrm{mg} / \mathrm{kg}$ & 65.5 & 5.9 \\
\hline Sulfur & wt:-\% & 1.89 & 2.02 \\
\hline HCl-extractable Ss sulfur & wt:\% & 0.29 & 1.04 \\
\hline Other sulfur forms & $w t=\%$ & 1.61 & 0.98 \\
\hline AVS & $\mathrm{g}(\mathrm{S}) / \mathrm{kg}$ & $4.3 \times 10^{-}=3$ & $9.4 \times 10^{-}=5$ \\
\hline NP & $\mathrm{kg}(\mathrm{CaCO}) / \mathrm{t}$ & 384 & 536 \\
\hline AP & $\mathrm{kg}(\mathrm{CaCO} 3) / \mathrm{t}$ & 50 & 31 \\
\hline Iron & $w t=\%$ & 3.7 & 3.4 \\
\hline Copper & $\mathrm{mg} / \mathrm{kg}$ & 1445 & 835 \\
\hline Lead & $\mathrm{mg} / \mathrm{kg}$ & 760 & 1260 \\
\hline Zinc & $\mathrm{mg} / \mathrm{kg}$ & 2085 & 2550 \\
\hline
\end{tabular}

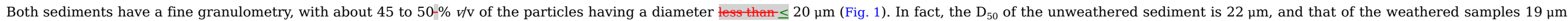

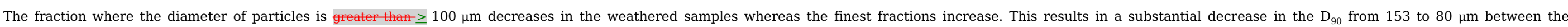
unweathered and weathered sediment. 


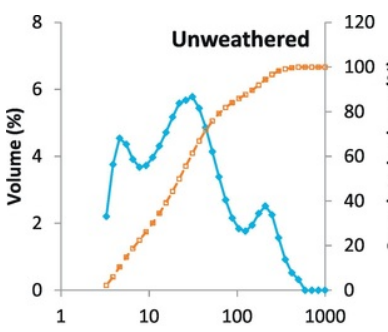

Diameter $(\mu \mathrm{m})$

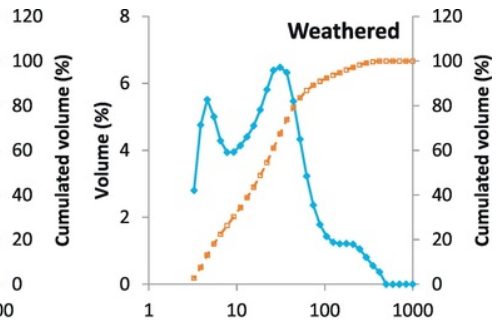

$\longrightarrow \%$ passer-by Diameter $(\mu \mathrm{m})$

a. $\quad$ b. $\quad \underset{--\infty}{-}-\frac{1}{-\infty}$ passer-by

Fig. 1 Particles size distribution by laser diffraction of unweathered (a) and weathered (b) sediments (\% in volume).

alt-text: Fig. 1

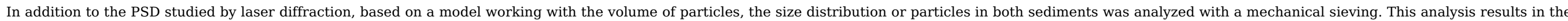
particle mass distribution, in order to determine the distribution of trace metals in the different fractions of unweathered (Fig. 2a) and weathered (Fig. 2b) sediments.
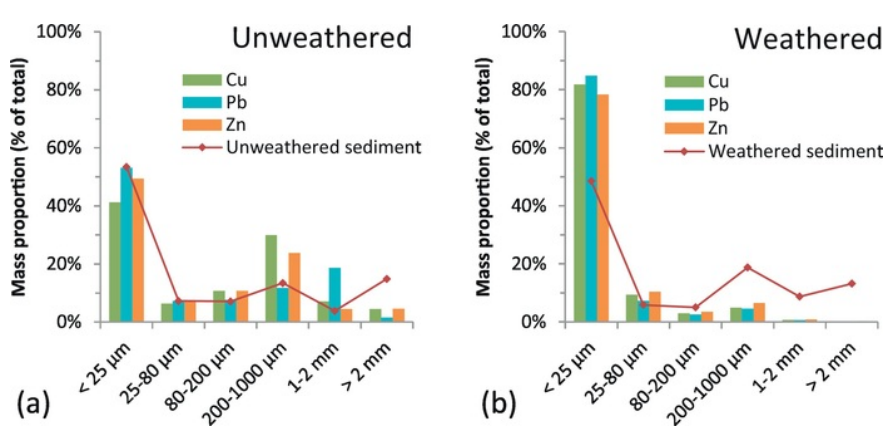
alt-text: Fig. 2

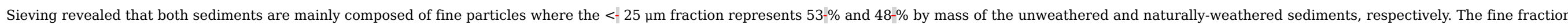

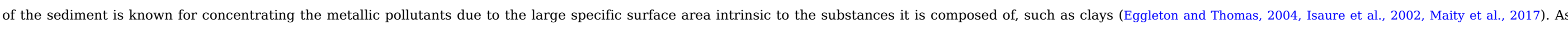

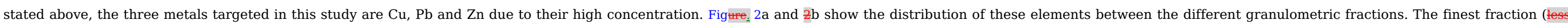

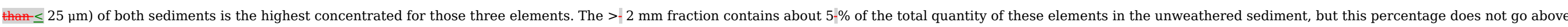

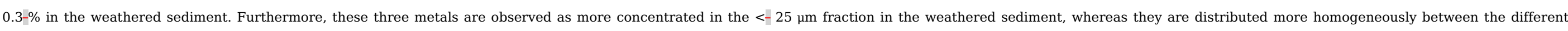

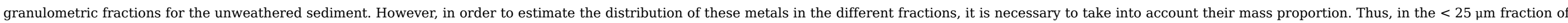

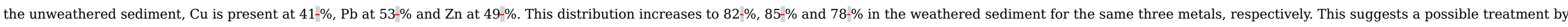

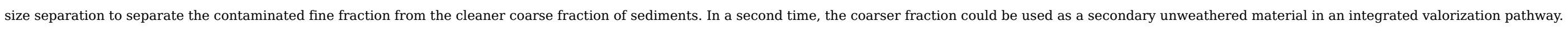

\section{2. $\underline{4.2}$ Mineralogical characterization}

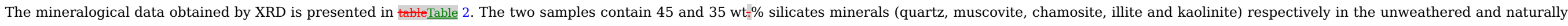

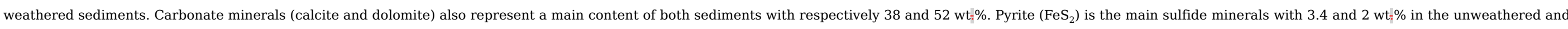

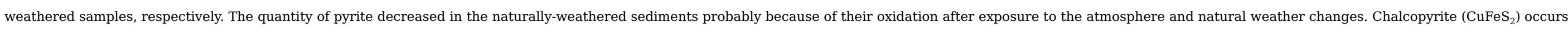

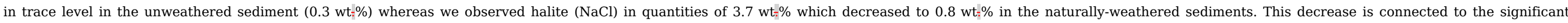


Table 2 XRD mineralogical analyses of unweathered and weathered sediments.

\section{alt-text: Table 2}

Mineral

Chemical formula

Composition (wt:\%)

\begin{tabular}{|c|c|c|c|c|}
\hline & & & \multirow[b]{2}{*}{ Unweath. } & \multirow[b]{2}{*}{ Weath. } \\
\hline & & & & \\
\hline \multirow[t]{5}{*}{ Silicates } & Quartz & $\mathrm{SiO}_{2}$ & 15.65 & 15.3 \\
\hline & Muscovite & $\mathrm{KAl}_{2}\left(\mathrm{Si}_{3} \mathrm{Al}\right) \mathrm{O}_{10}(\mathrm{OH}, \mathrm{F})_{2}$ & $<-\mathrm{LDM}$ & 9.24 \\
\hline & Chamosite & $(\mathrm{Fe}, \mathrm{Mg})_{5} \mathrm{Al}\left(\mathrm{Si}_{3} \mathrm{Al}\right) \mathrm{O}_{10}(\mathrm{OH}, \mathrm{O})$ & 6.5 & 4.47 \\
\hline & Illite & $\left(\mathrm{K}, \mathrm{H}_{3} \mathrm{O}\right)(\mathrm{Al}, \mathrm{Mg}, \mathrm{Fe})_{2}(\mathrm{Si}, \mathrm{Al})_{4} \mathrm{O}_{10}\left[(\mathrm{OH})_{2},\left(\mathrm{H}_{2} \mathrm{O}\right)\right]$ & 13.2 & 1.55 \\
\hline & Kaolinite & $\mathrm{Al}_{2} \mathrm{Si}_{2} \mathrm{O}_{5}(\mathrm{OH})_{4}$ & 9.8 & 4 \\
\hline \multirow[t]{2}{*}{ Carbonates } & Dolomite & $\mathrm{CaMg}\left(\mathrm{CO}_{3}\right)_{2}$ & 6.8 & 13.6 \\
\hline & Calcite & $\mathrm{CaCO}_{3}$ & 31.05 & 38.9 \\
\hline \multirow[t]{2}{*}{ Sulfides } & Pyrite & $\mathrm{FeS}_{2}$ & 3.45 & 1,98 \\
\hline & Chalcopyrite & $\mathrm{CuFeS}_{2}$ & 0.33 & $<\mathrm{LDM}$ \\
\hline \multirow[t]{3}{*}{ Other minerals } & Halite & $\mathrm{NaCl}$ & 3.75 & 0.76 \\
\hline & Gypsum & $\mathrm{CaSO}_{4 \div-} \div 2 \mathrm{H}_{2} \mathrm{O}$ & $<\mathrm{LDM}$ & 5 \\
\hline & Magnetite & $\mathrm{Fe}_{3} \mathrm{O}_{4}$ & 0.2 & 1.1 \\
\hline
\end{tabular}

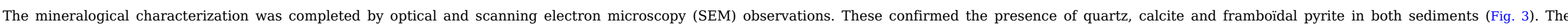

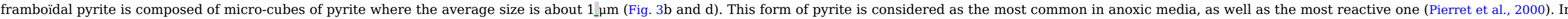

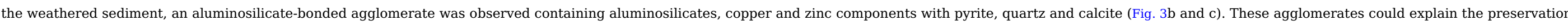

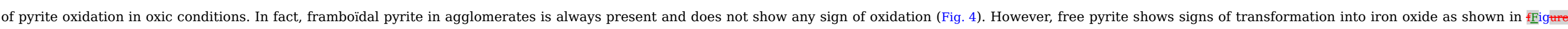
$4 \mathrm{a}$ and $\mathrm{b}$. In the unweathered sediment, pyrrhotite $\left(\mathrm{Fe}_{1-\mathrm{x}} \mathrm{S}\right)$ was observed and shows signs of transformation into hematite $\left(\mathrm{Fe}_{2} \mathrm{O}_{3}\right)$ and magnetite $\left(\mathrm{Fe}_{3} \mathrm{O}_{4}\right)(\mathrm{Fig}$. $4 \mathrm{c}$ and d). 

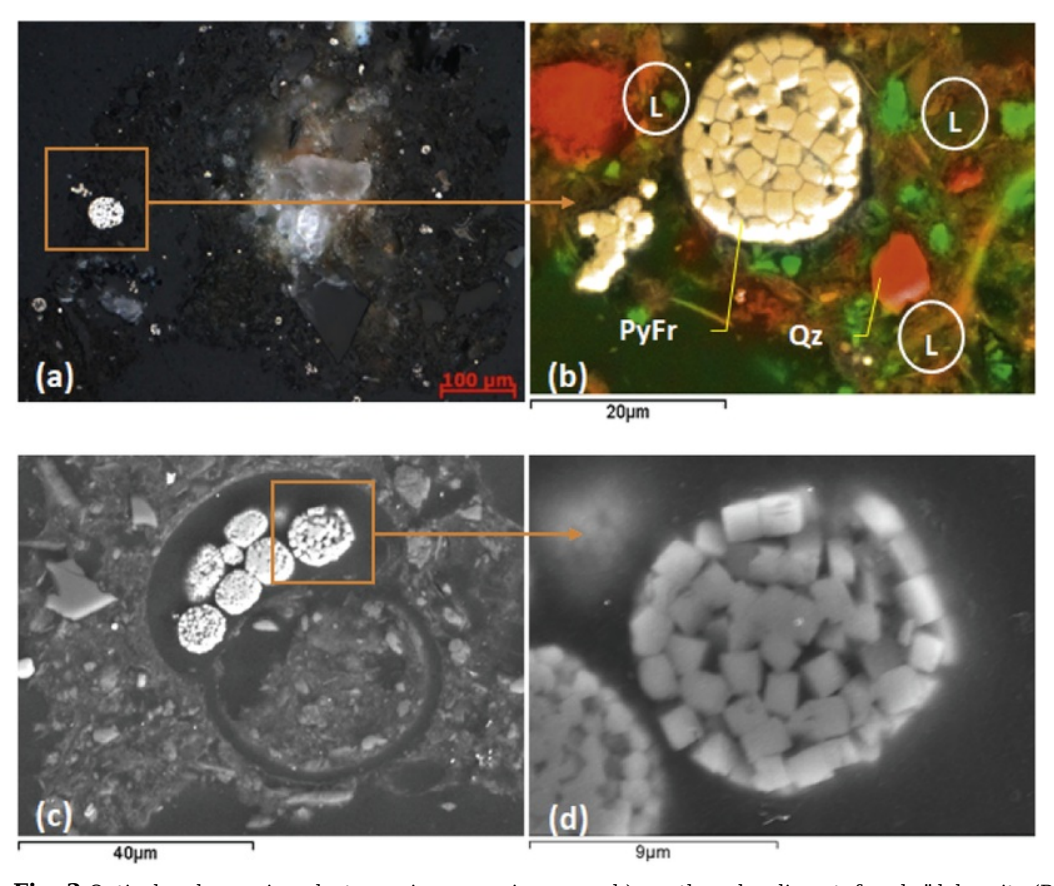

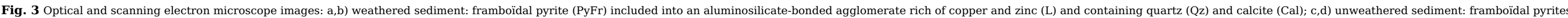
alt-text: Fig. 3

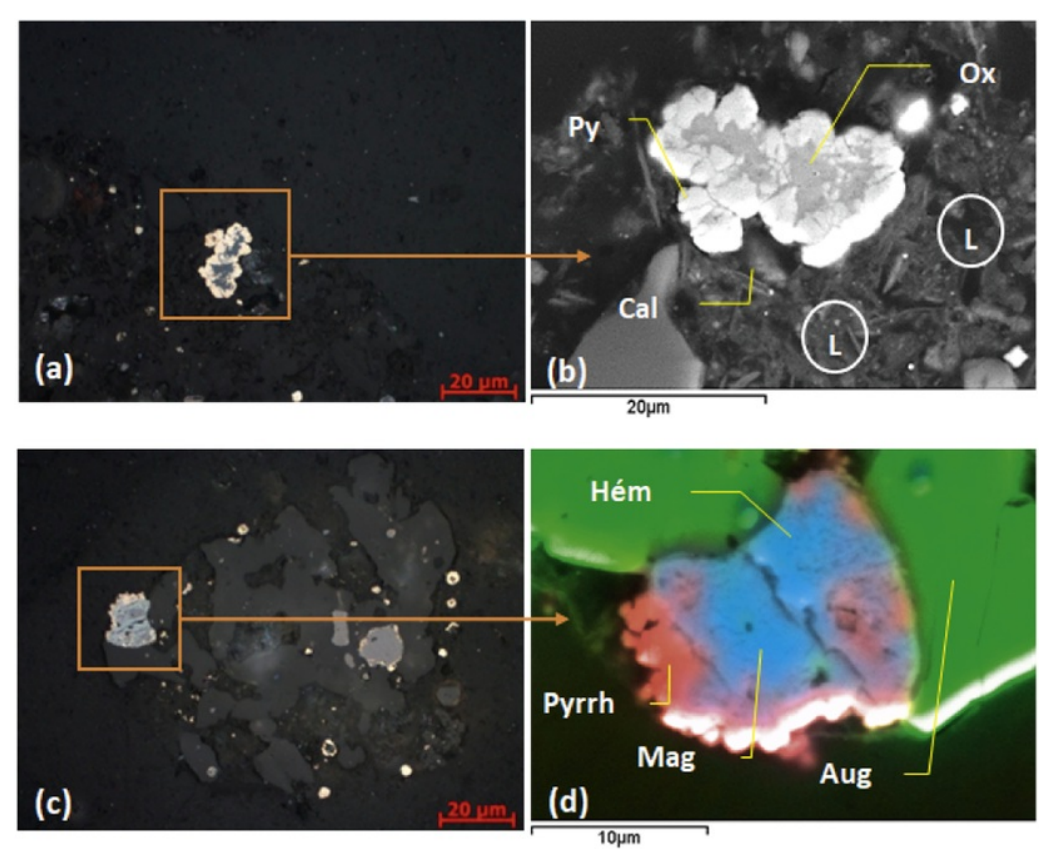

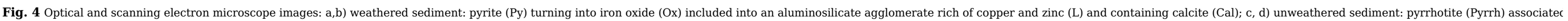




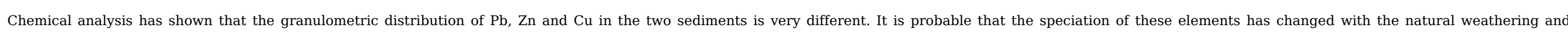

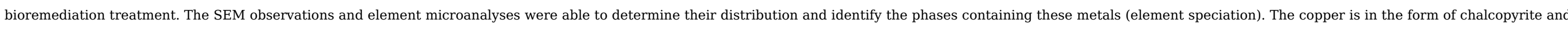

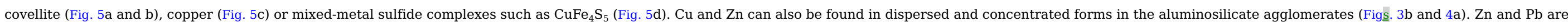
not found in any crystallized mineral form. Figure, 6 illustrates the X-ray mapping of Zn showing its association to sulfur distribution, but not occurring as sulfide minerals.

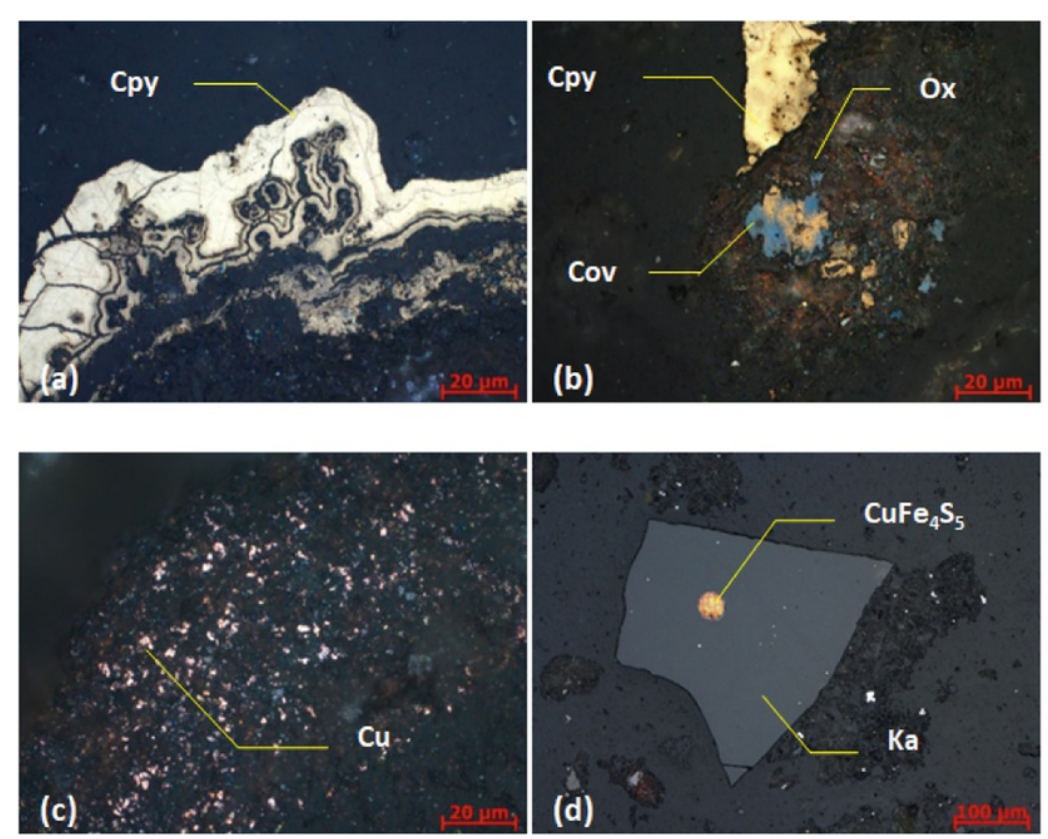

Fig. 5 Optical microscope images of unweathered sediment: a, b) chalcopyrite (Cpy) and covellite (Cov); c) native copper (Cu); d) iron and copper mixed sulfide CuFe4S5 included into Katophorite (Ka). alt-text: Fig. 5 

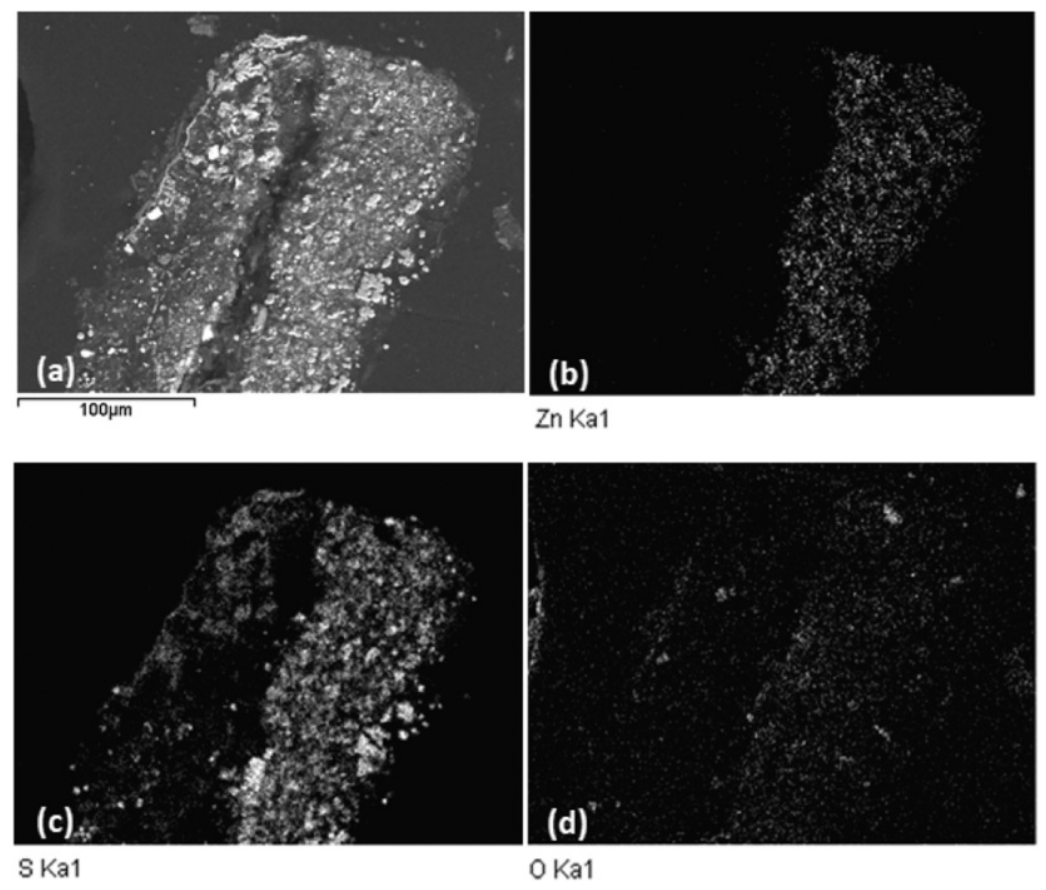

Fig. 6 a) Scanning electron microscope image of a grain in unweathered sediment; b, c, d) X-mapping images showing zinc, sulfur and oxygen distribution in the grain of unweathered sediment.

alt-text: Fig. 6

\subsubsection{Leaching tests in weathering cells}

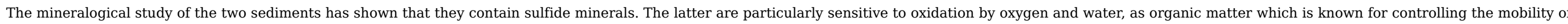

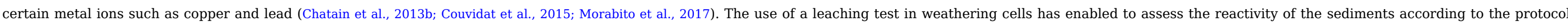
described previously.

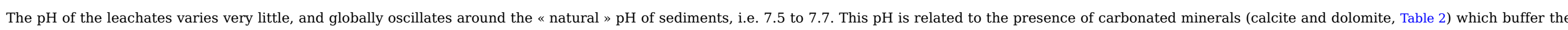

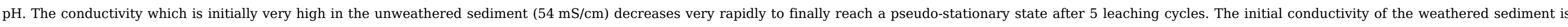

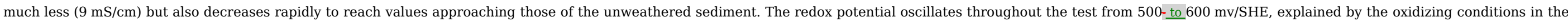
two sediments.

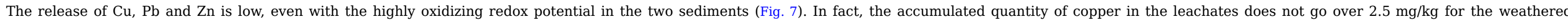

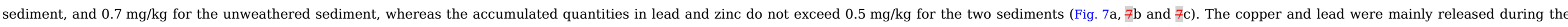

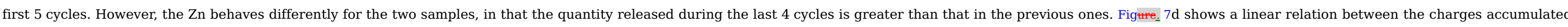

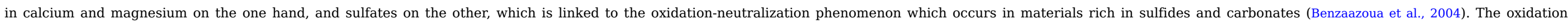

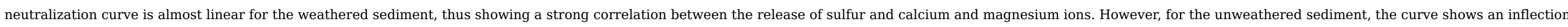
during the first 4 cycles before becoming linear, which might mean that release of S, Ca and Mg into the lixiviates is probably partially disconnected. 

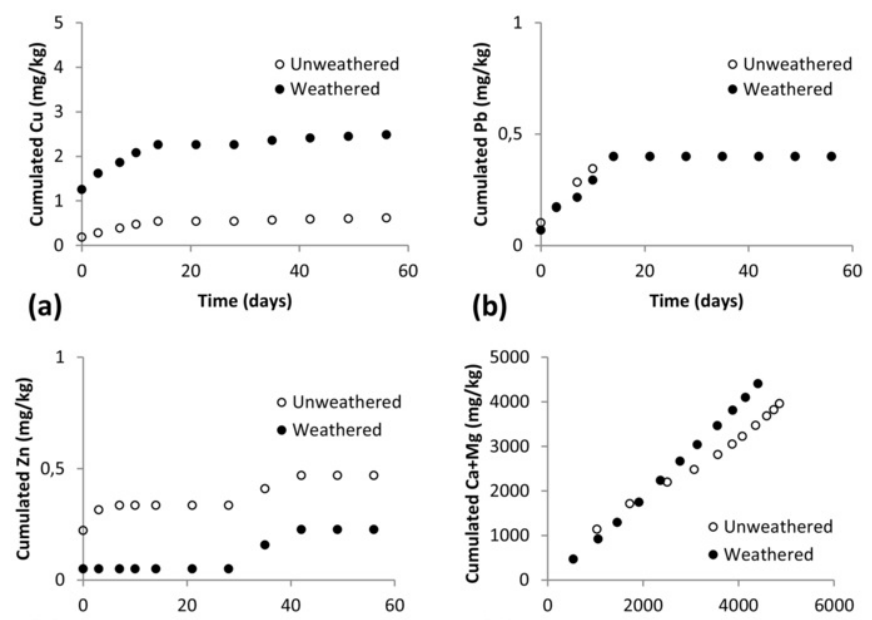

(b)

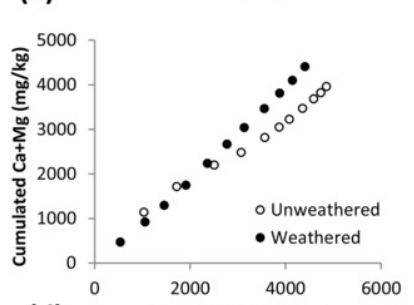

(c)

Time (days)

(d)

$2000 \quad 4000$
Cumulated $S(\mathrm{mg} / \mathrm{kg})$

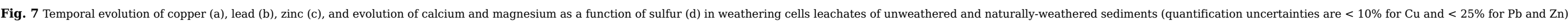
alt-text: Fig. 7

\section{5. $\underline{5}$ Discussion}

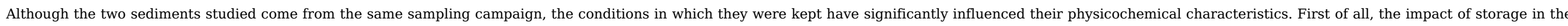

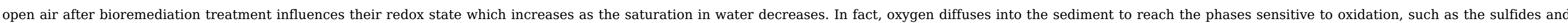

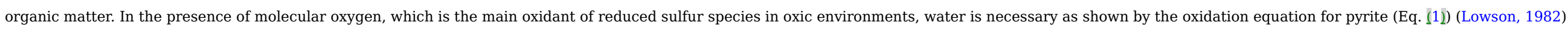

$2 \mathrm{FeS}_{2}+7 \mathrm{O}_{2}+2 \mathrm{H}_{2} \mathrm{O} \rightarrow 2 \mathrm{Fe}^{2+}+4 \mathrm{SO}_{4}^{2-}+4 \mathrm{H}^{+}$

The atmospheric oxygen combined to the meteoritic water enables oxidation at the same time as the leaching of most of the mobile elements such as chlorides and oxidation products such as sulfates.

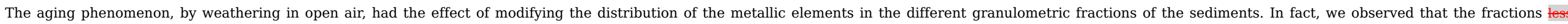

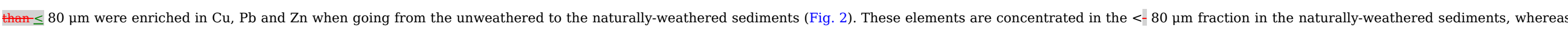

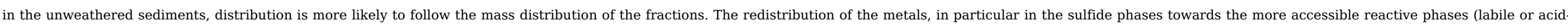
soluble), was also observed after aeration and drying of the sediment (Claff et al., 2010; Calmano et al., 1993).

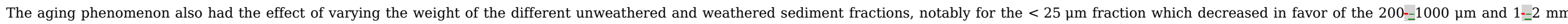

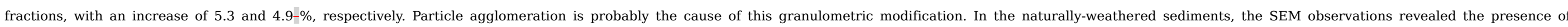

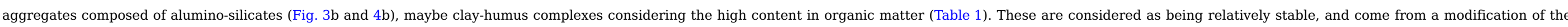

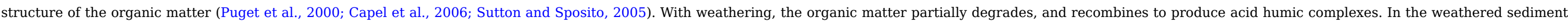

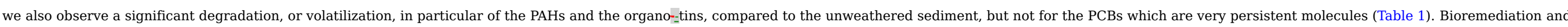

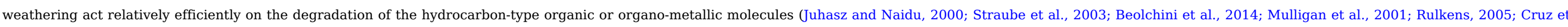
al., 2017).

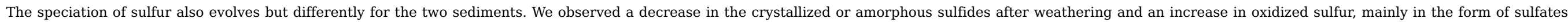

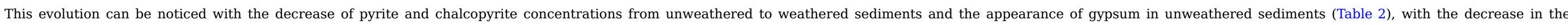

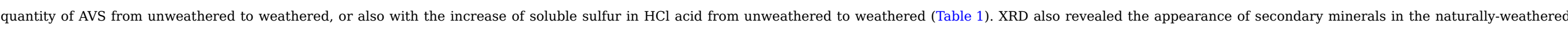




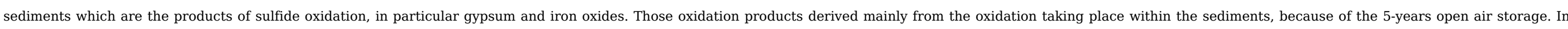

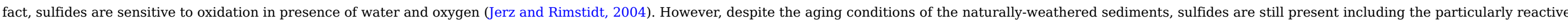

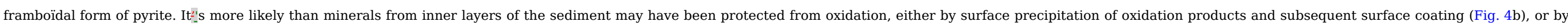

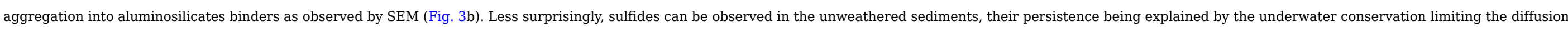
of oxygen in the sedimentary layers.

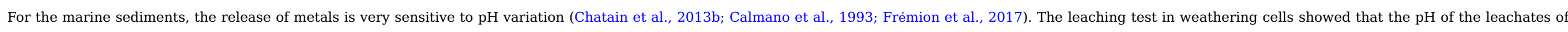

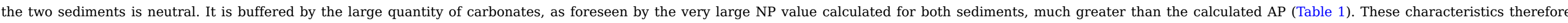

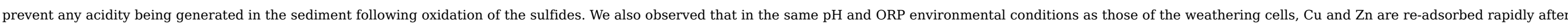

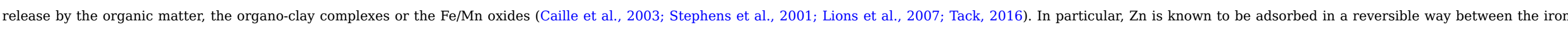

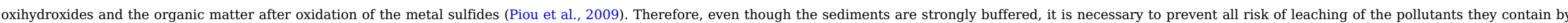

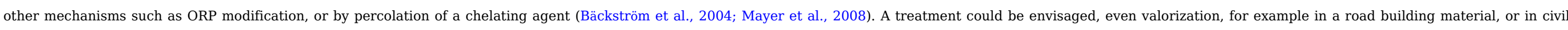
engineering by treatment with hydraulic binders (Paria and Yuet, 2006; Pinto et al., 2011; Wang et al., 2012; Couvidat et al., 2016a; Bao et al., 2016; Saussaye et al., 2016).

\section{6. $\underline{6}$ Conclusion}

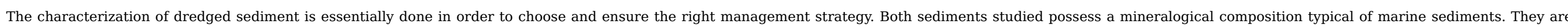

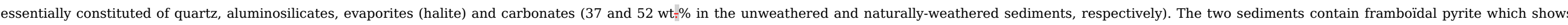

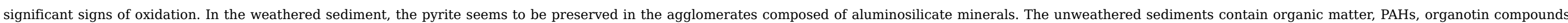

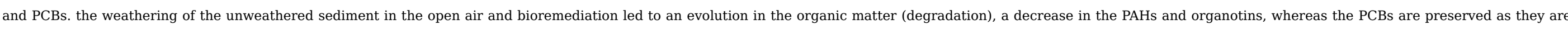
more persistent.

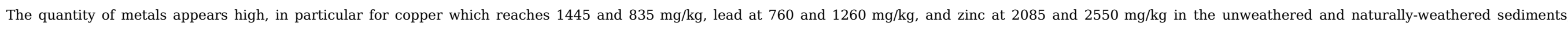

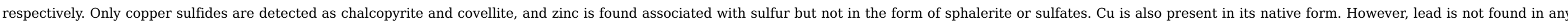

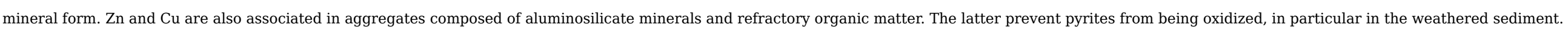

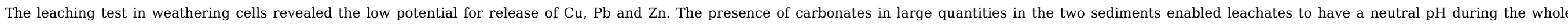

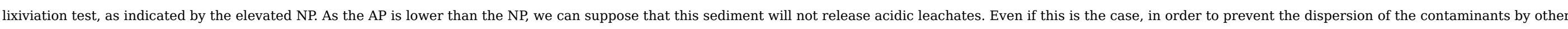
possible mechanisms, a treatment and/or valorization could be envisaged, in civil engineering or the road-building domain, for example (Couvidat et al., 2016a; Saussaye et al., 2016; Bao et al., 2016).

\section{Acknowledgments}

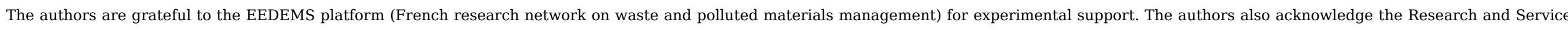
Unit in Mineral Technology (URSTM), University of Quebec in Abitibi-Temiscamingue (UQAT) for their experimental support.

\section{References}

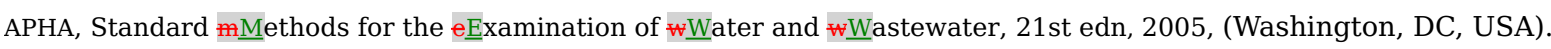

Bäckström M., Karlsson S., Bäckman L., Folkeson L. and Lind B., Mobilisation of heavy metals by deicing salts in a roadside environment,

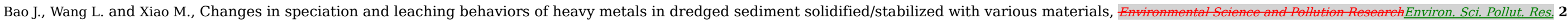
(9), 2016, 8294-8301.

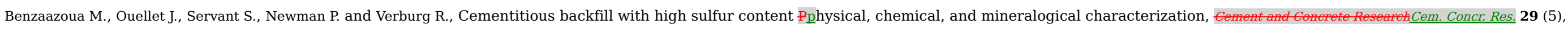
1999, 719-725. 


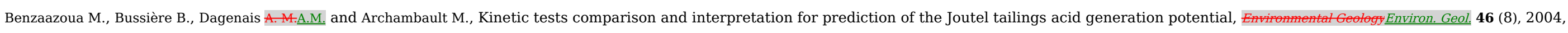
1086-1101.

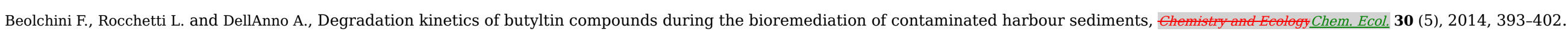

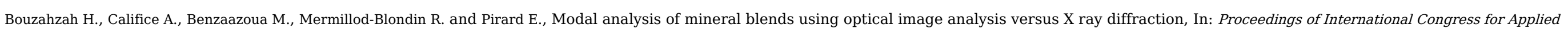
Mineralogy ICAMO8, Brisbane, Australia: AusIMM, 2008.

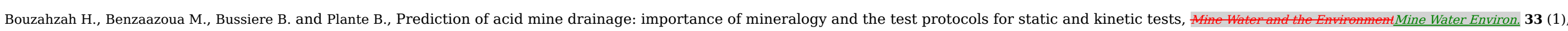
2014, 54-65.

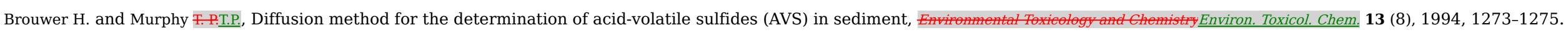

Caille N., Tiffreau C., Leyval C. and Morel J.L.J.L., Solubility of metals in an anoxic sediment during prolonged aeration, Seiee of The Total EnvironmentSci. Total Environ. 301 (1-3), 2003, 239-250.

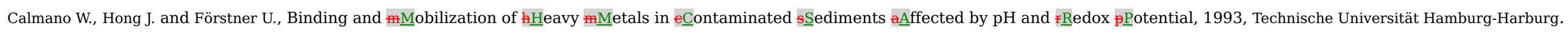

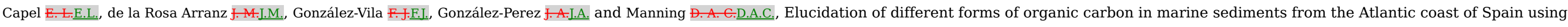

thermal analysis coupled to isotope ratio and quadrupole mass spectrometry, Erganic Geochistryorg. Geochem. 37 (12), 2006, 1983-1994.

Caplat C., Texier H., Barillier D. and Lelievre C., Heavy metals mobility in harbour contaminated sediments: Fthe case of Port-en-Bessin, Marrine Pollution ButletimMar. Pollut. Bull. 50 (5), $2005,504-511$.

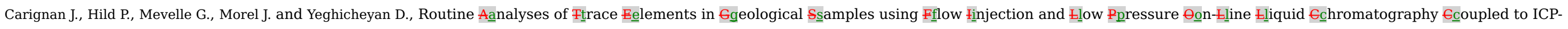

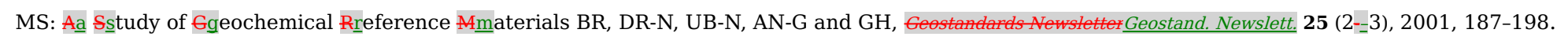

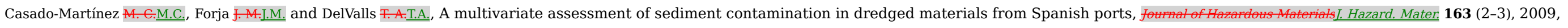
1353-1359.

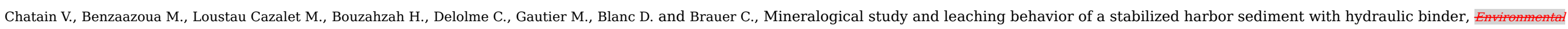
Science and Pollution ResearchEnviron. Sci. Pollut. Res. 2013a, 1-9.

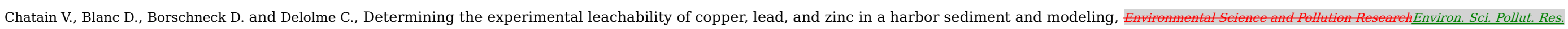
2013b, 1-9.

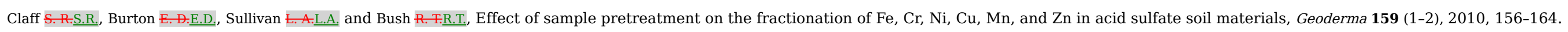

Commission Decision of 3 May 2000 Replacing Decision 94/3/EC Establishing a List of Wastes, Consolidated Version of 01 January, $2000 D 0532,2002$.

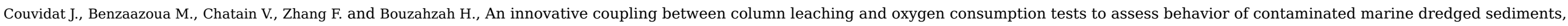
Enviromental Seience and Pollution ResearchEnviron. Sci. Pollut. Res. 2015, 1-13.

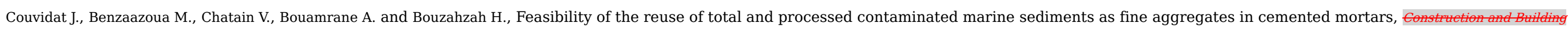
Materials Constr. Build. Mater. 112, 2016a, 892-902.

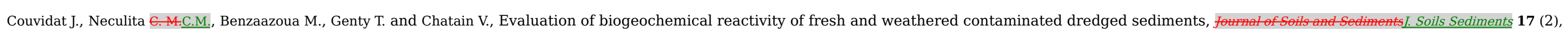
2016b, 543-556.

Cruz R., Méndez B.A.B.A., Monroy M. and González I., Cyclic voltammetry applied to evaluate reactivity in sulfide mining residues, Applied GeochemistryAppl. Geochem. 16 (14), 2001, 1631-1640.

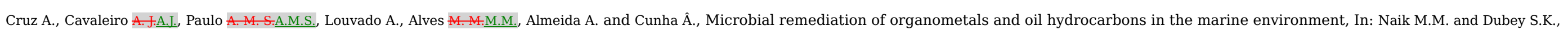


(Eds.), Marine Pollution and Microbial Remediation, 2017, Singapore; Springer Singapore, 41-66.

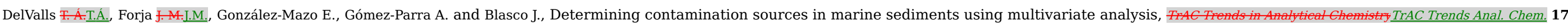

(4), 1998, 181-192.

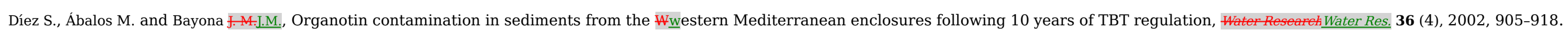

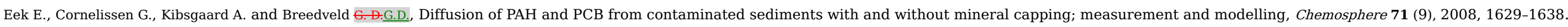

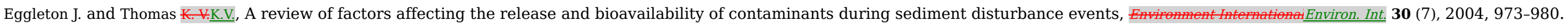

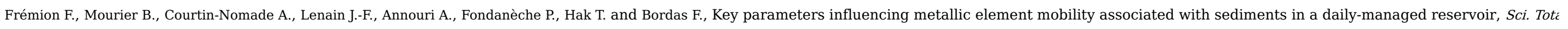
Environ. 605-606 (Supplement C), 2017, 666-676.

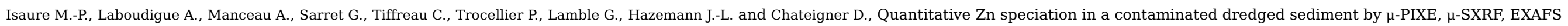
spectroscopy and principal component analysis,

Jerz J.K.J.K. and Rimstidt J. P.J.D., Pyrite oxidation in moist air,

Jones B. and Turki A., Distribution and speciation of heavy metals in surficial sediments from the Tees Estuary, north-east England, Marine Pollution Bulletimar. Pollut. Bull. 34 (10), 1997, 768-779.

JORF 2007, 17002, n²40 du 16 octobre. (texte $\mathrm{n}^{\circ} 1$ ).

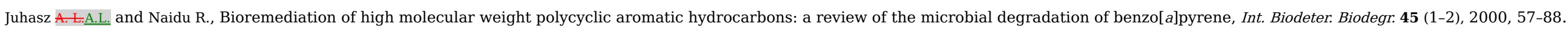

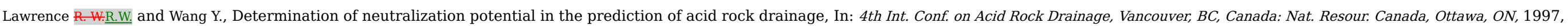

$451-463$.

Le Guyader C., Enquête “Dragage 2009” - Synthèse des données [Report], Margny Lès Compiègne: CETMEFEQ-CETMEF-12-05-FR, 2011.

Le Guyader C., Enquête “Dragage 2010” - Synthèse des données [Report], Margny Lès Compiègne: CETMEFEQ-CETMEF-13-06-FR, 2013.

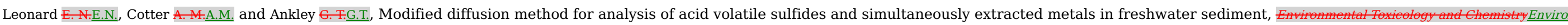
Toxicol. Chem. 15 (9), 1996, 1479-1481.

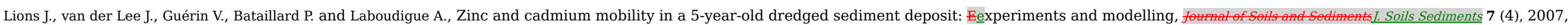
207-215.

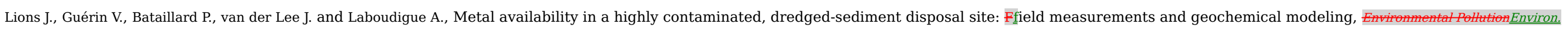
Pollut. 158 (9), 2010, 2857-2864.

Lowson R. T.R.T., Aqueous oxidation of pyrite by molecular oxygen, themieal Reviews Chem. Rev 82 (5), 1982, 461-497.

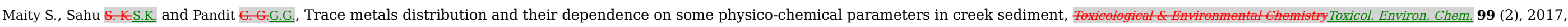
209-222.

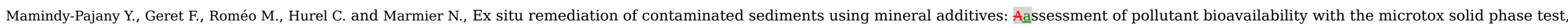
Chemosphere 86 (11), 2012, 1112-1116.

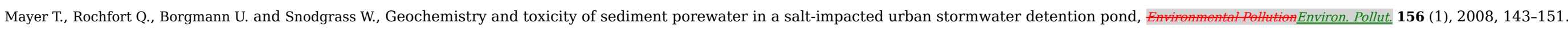




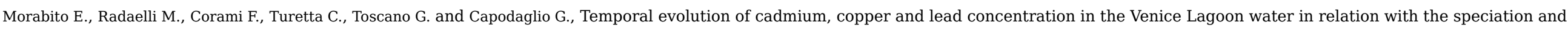
dissolved/particulate partition, Mar. Pollut. Bull. 2017.

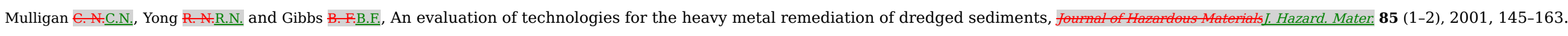

Paktunc A. D.A.D., Characterization of mine wastes for prediction of acid mine drainage, In: Environmental Impacts of Mining Activities, $1999 \mathrm{a}$, Springer, $19-40$.

Paktunc A. D.A.D., Mineralogical constraints on the determination of neutralization potential and prediction of acid mine drainage, Envirmental Geogy Environ. Geol. 39 (2), 1999b, 103-112.

Paria S. and Yuet P. K.P.K., Solidification-stabilization of organic and inorganic contaminants using portland cement: a literature review, Environmental ReviewsEnviron. Rev 14 (4), 2006 , 217-255.

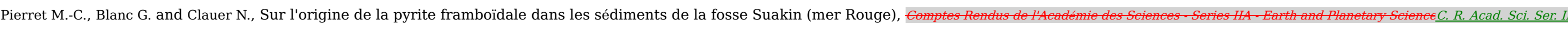
Earth Planet. SCi. 330 (1), 2000, 31-38.

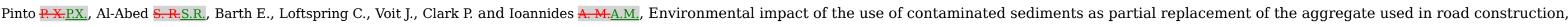
foumal of Hazardous Materials.j. Hazard. Mater, 189 (1-2), 2011, 546-555.

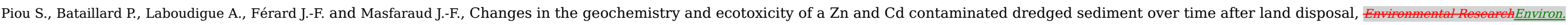
Res. 109 (6), 2009, 712-720.

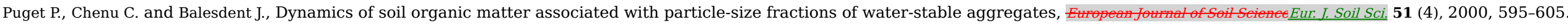

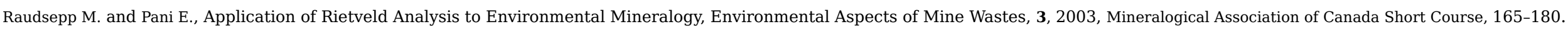

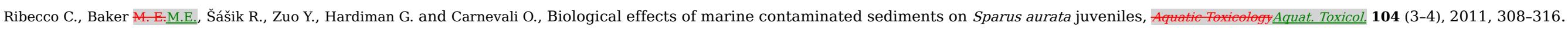

Rickard D. and Morse J.W.J.W., Acid volatile sulfide (AVS), Marine Chem 97 (3-4), 2005, 141-197.

Rulkens W., Introduction to the Ftreatment of Ppolluted Ssediments, Revis

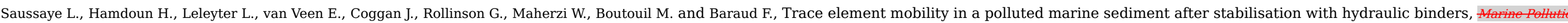
Bulletim Mar. Pollut. Bull. 110 (1), 2016, 401-408.

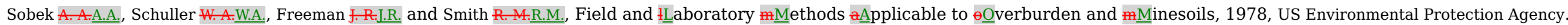

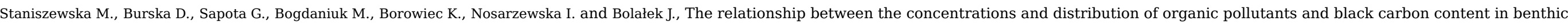
sediments in the Gulf of Gdańsk, Baltic Sea, Marine Pollution BulletimMar. Pollut. Bull. 62 (7), 2011, 1464-1475.

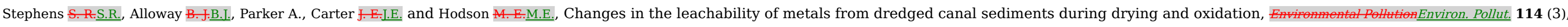
2001, 407-413.

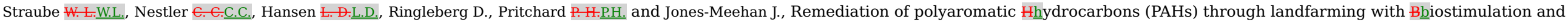

Bbioaugmentation, Acta BiotechnologieaActa Biotechnol. 23 (2--3), 2003, 179-196.

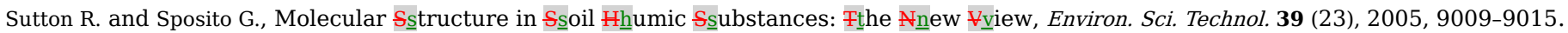

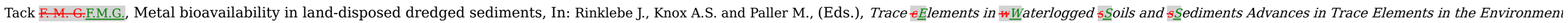
2016, CRC Press; Boca Raton, FL, USA, 253-266.

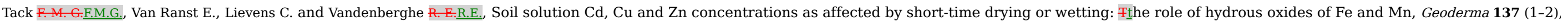


2006, 83-89

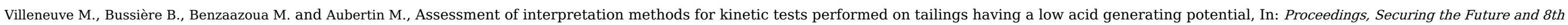
ICARD, Skelleftea, Sweden, 2009.

Wang D. X.D.X., Abriak N.E.N.E., Zentar R. and Xu W., Solidification/stabilization of dredged marine sediments for road construction, Envirommental technologf Environ. Technol. 33 (1), 2012 , 95-101.

Young P.A.R.A., The Rietveld Method, 1993, Oxford University Press; NYC, NY, USA.

\section{Graphical abstract}

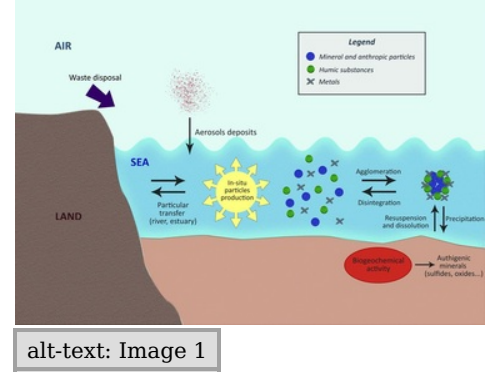

Highlights

- In-depth study of contaminated sediments is advised for accurate management.

- Reactive mineral species in particular framboïdal pyrite were observed in materials.

- The natural weathering has led to a significant decrease in PAHs and organotins.

- The leaching test revealed the low potential for release of $\mathrm{Cu}, \mathrm{Pb}$ and $\mathrm{Zn}$.

- Size separation and/or a valorization in civil engineering could be envisaged.

\section{Queries and Answers}

Query:

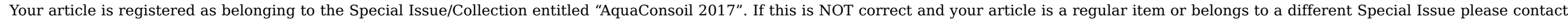
p.das@elsevier.com immediately prior to returning your corrections.

Answer: Yes

Query:

Please confirm that given names and surnames have been identified correctly and are presented in the desired order, and please carefully verify the spelling of all authors' names. 
Answer: Yes

Query:

The author names have been tagged as given names and surnames (surnames are highlighted in teal color). Please confirm if they have been identified correctly

Answer: Yes

Query:

Please check and confirm if the authors' affiliations are presented correctly and amend as necessary.

Answer: I confirm that it is correct.

Query:

One parenthesis has been deleted to balance the delimiters. Please check and confirm if this was done correctly.

Answer: Yes

Query:

The data "IRME" has been deleted to avoid redundancy. Please check if the deletion is appropriate and amend as necessary.

Answer: I confirm that it is correct.

Query:

Please check the hierarchy of the section headings and confirm if correct.

Answer: Yes

Query:

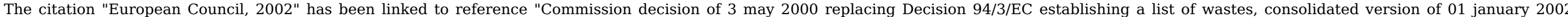
(2000D0532)". Please check if appropriate and amend as necessary.

Answer: I confirm that it is correct.

Query:

The citation "French Official Journal, 2007" has been linked to reference "JORF n²40 du 16 octobre 2007 p.17002 texte n". Please check if appropriate and amend as necessary. Answer: I confirm that it is correct.

Query:

The citation "Sobek (1978)" has been changed to "Sobek et al. (1978)" to match the author name/date in the reference list. Please check if the change is fine and amend if necessary.

Answer: I confirm that it is correct.

Query:

Please check if the insertion of "2008" in this reference item is correct and amend as necessary. 
Answer: I confirm that it is correct.

Query:

Please check if the insertion of "1997" in this reference item is correct and amend as necessary.

Answer: I confirm that it is correct.

Query:

Please provide the volume number and page range for the bibliography in Morabito et al., 2017

Answer: In press, https://doi.org/10.1016/j.marpolbul.2017.10.043 